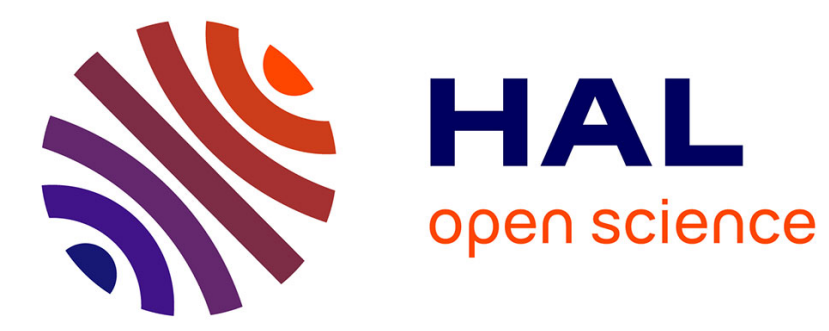

\title{
Interaction of spin-density waves with defects and quasiparticles
}

\author{
G. Kriza, O. Traetteberg
}

\section{To cite this version:}

G. Kriza, O. Traetteberg. Interaction of spin-density waves with defects and quasiparticles. Journal de Physique IV Proceedings, 1993, 03 (C2), pp.C2-15-C2-20. 10.1051/jp4:1993203 • jpa-00251572

\section{HAL Id: jpa-00251572 https://hal.science/jpa-00251572}

Submitted on 1 Jan 1993

HAL is a multi-disciplinary open access archive for the deposit and dissemination of scientific research documents, whether they are published or not. The documents may come from teaching and research institutions in France or abroad, or from public or private research centers.
L'archive ouverte pluridisciplinaire HAL, est destinée au dépôt et à la diffusion de documents scientifiques de niveau recherche, publiés ou non, émanant des établissements d'enseignement et de recherche français ou étrangers, des laboratoires publics ou privés. 


\title{
Interaction of spin-density waves with defects and quasiparticles
}

\author{
G. KRIZA and O. TRAETTEBERG \\ Laboratoire de Physique des Solides, URA 2 CNRS, Université Paris-Sud, 91405 Orsay, France
}

\begin{abstract}
The results of recent transport measurements in the spin-density wave (SDW) state of the Bechgaard salts $(\mathrm{TMTSF})_{2} \mathrm{X}\left[\mathrm{X}=\mathrm{PF}_{6}, \mathrm{AsF}_{6}, \mathrm{SbF}_{6}\right.$, or $\left.\left(\mathrm{AsF}_{6}\right)_{(1-x)}\left(\mathrm{SbF}_{6}\right)_{x}\right]$ are summarized. The weak nonmagnetic disorder introduced by the alloying of two kinds of anions increases the depinning threshold by up to 3 orders of magnitude. The damping of the low-frequency phase mode decreases exponentially with increasing temperature and saturates at a constant value determined by the disorder at high temperatures. The dc nonlinear conductivity disappears at low temperatures, and this behavior is not explained by the increasing damping. From Hall effect measurements we find a strong coupling between the SDW and normal electrons.
\end{abstract}

\section{Introduction}

In the density wave (DW) ground states [charge-density wave (CDW) and spin-density wave (SDW)] the translational symmetry of the electronic system is broken by the formation of a static, periodic modulation of the electronic density for each spin direction [1,2]. The immediate consequence of the broken translational symmetry is that the DW interacts with the defects of the crystal lattice. Moreover, because the long range order is diagonal, rather than off-diagonal like in superconductors, the fluctuations of the phase of the DW order parameter correspond to charge fluctuations, consequently the Coulomb effects are very different in DW's and superconductors. The two fundamental consequences of these interactions are the pinning of the phase of the DW to the crystal lattice and the damping of the phase excitations.

Both pinning and damping can be studied by transport measurements. The current-voltage characteristics become nonlinear at a threshold electric field which is directly related to the pinning force. An alternative measure of the pinning force is the inverse of the static dielectric constant. The damping of the lowfrequency phase excitations are characterized by the mean dielectric relaxation rate, or by the excess dc conductivity in a given electric field above the depinning threshold.

In this paper we review the results of extensive transport measurements in the only model systems of collective SDW response, the Bechgaard salts (TMTSF) ${ }_{2} \mathrm{PF}_{6}\left[\mathrm{X}=\mathrm{PF}_{6}, \mathrm{AsF}_{6}\right.$, etc. $]$. The effect of disorder is studied in the solid solutions (TMTSF $)_{2}\left(\mathrm{AsF}_{6}\right)_{(1-x)}\left(\mathrm{SbF}_{6}\right)_{x}$. Although this weak nonmagnetic disorder has no significant effect on the metallic transport, it dominates the properties of the collective-mode SDW transport: the depinning threshold increases by up to 3 orders of magnitude. We observe a crossover in the SDW damping from a temperature-independent region at high temperatures to an exponentially temperature-dependent low-temperature region. As seen by Hall effect measurements, when the SDW is depinned, the transport by the thermally excited quasiparticles also becomes nonlinear as a function of the electric field. We discuss these findings in terms of the interaction of the SDW with defects and thermally excited quasiparticles.

\section{SDW pinning}

For CDW's, it is well established that the main source of pinning is the interaction of the DW with point defects of the crystal lattice [1]. The direct analogue of this pinning for the SDW case is the pinning by 


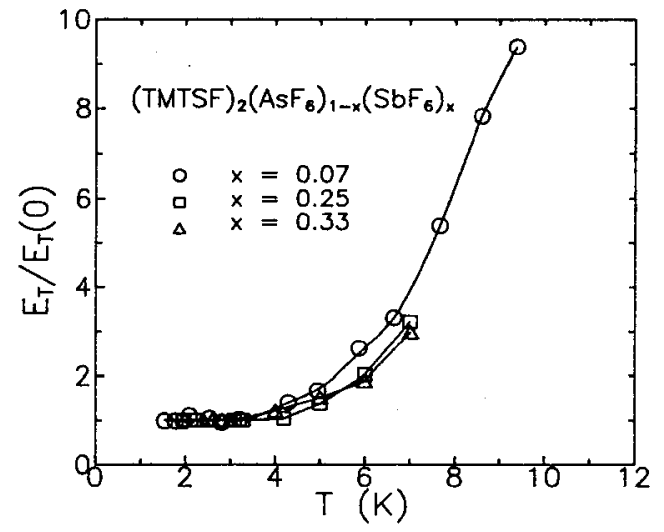

FIG. 1

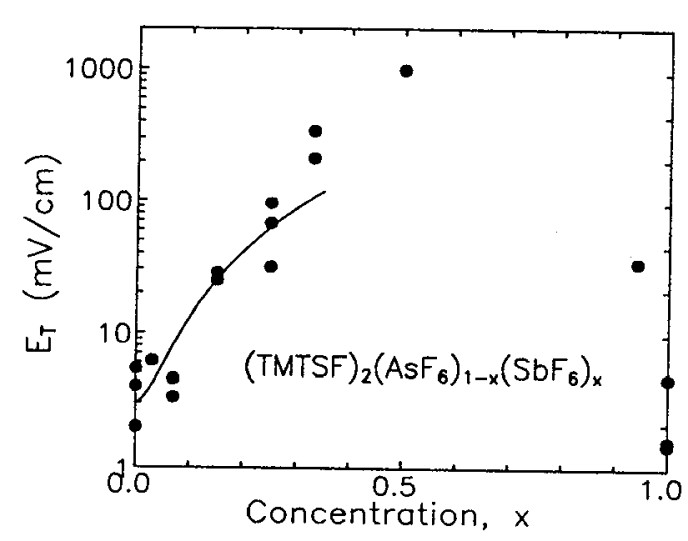

FIG. 2

FIG. 1 Threshold field for SDW depinning as a function of temperature, normalized to the low-temperature value for various members of the series $(\mathrm{TMTSF})_{2}\left(\mathrm{AsF}_{6}\right)_{(1-x)}\left(\mathrm{SbF}_{6}\right)_{x}$.

FIG. 2 Low-temperature threshold field as a function of the $\mathrm{SbF}_{6}$ concentration in the series $(\mathrm{TMTSF})_{2}\left(\mathrm{AsF}_{6}\right)_{(1-x)}\left(\mathrm{SbF}_{6}\right)_{x}$.

localized magnetic moments. However, it has been predicted [3] that nonmagnetic defects also pin the phase of the SDW and this mechanism is expected to be dominant.

A very strong perturbation of the conducting chains is the destruction of the TMTSF molecule by highenergy irradiation. The effect of radiation defects on the depinning threshold of SDW's in (TMTSF) ${ }_{2} \mathrm{PF}_{6}$ has been studied recently by Kang et al. [4], and-in agreement with the expectations - they find a strong linear increase of the threshold field with the defect concentration. The opposite limit, probably the weakest controllable disorder in any density wave system is realized by the solid solution of two kinds of isoelectronic, isosteric anions with slightly different size in the series $(\mathrm{TMTSF})_{2}\left(\mathrm{AsF}_{6}\right)_{(1-x)}\left(\mathrm{SbF}_{6}\right)_{x}[5,6]$. Moreover, in contrast to the radiation defects which are known to be magnetic [7], the anion disorder is not expected to create localized magnetic moments.

The temperature dependence of the threshold field $E_{T}$ for samples with 3 different $\mathrm{SbF}_{6}$ concentrations is shown in Fig. 1. The threshold field decreases with decreasing temperature, and saturates at about $4 \mathrm{~K}$, i.e., $1 / 3$ of the SDW transition temperature $T_{c}=12 \mathrm{~K}$, in qualitative agreement with previous findings [8] and with the generalized Fukuyama-Lee-Rice theory of Maki and Virosztek [9]. Figure 2 shows the threshold field as a function of the $\mathrm{SbF}_{6}$ concentration $x . E_{T}$ is the same in the "pure" systems $x=0,1$, but up to 3 orders of magnitude higher for intermediate $x$ values. The fact that $E_{\tau}$ has the same temperature dependence for all members of the series indicates that the mechanism of pinning does not change with increasing disorder. It is clear from Fig. 2 that the dependence of $E_{T}$ on the defect concentration is superlinear; the large scatter in the data, however, does not allow to infer a power-law exponent.

In conclusion, we have demonstrated a strong increase of $E_{T}$ due to weakly perturbing nonmagnetic point defects.

\section{SDW damping}

We have charaterized the low-frequency SDW damping $\gamma$ both by the nonlinear dc conductivity and by the low-frequency dielectric response as a function of temperature and disorder in the series $(\mathrm{TMTSF})_{2}\left(\mathrm{AsF}_{6}\right)_{(1-x)}\left(\mathrm{SbF}_{6}\right)_{x} \quad[5,6]$. From the nonlinear conductivity, we define the quantity $\sigma_{\mathrm{SDW}}^{\mathrm{dc}}=\sigma\left(2 E_{T}\right)-\sigma_{n} \propto \gamma^{-1}$, where $\sigma_{n}$ is the low-field conductivity, and from the dielectric response $\sigma_{\mathrm{SDW}}^{\mathrm{ac}}=2 \pi \varepsilon(0) / \tau \propto \gamma^{-1}$, where $\varepsilon(0)$ is the static dielectric constant and $\tau$ is the mean dielectric relaxation time. 


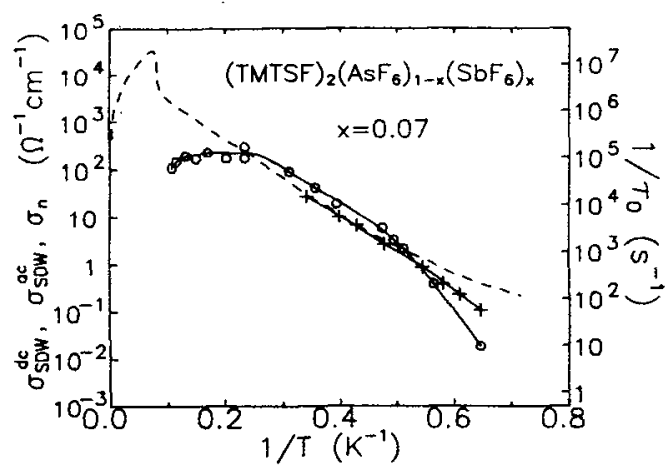

FIG. 3

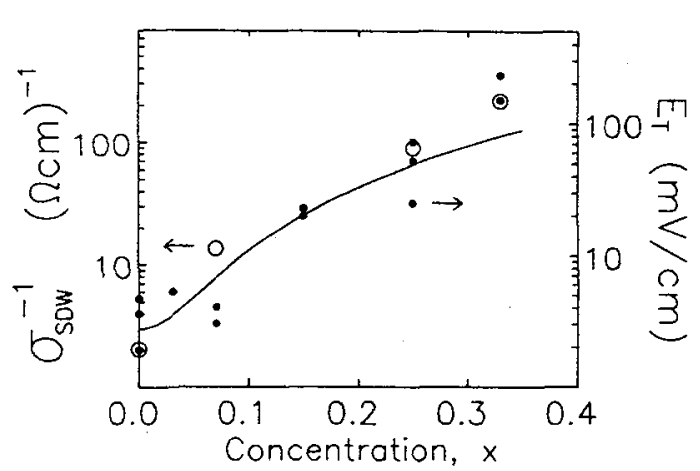

FIG. 4

FIG. 3 Arrhenius plot of the normal conductivity $\sigma_{n}$ (dashed line), together with the SDW conductivity inferred from the nonlinear dc conductivity, $\sigma_{\mathrm{SDW}}^{\mathrm{dc}}$ (circles) and from the low-frequency dielectric response, $\sigma_{\mathrm{SDW}}^{\mathrm{ac}}$ (crosses)

FIG. 4 The high-temperature SDW conductivity (large circles) together with the depinning threshold (filled small circles) as a function of the $\mathrm{SbF}_{6}$ concentration in the series $(\mathrm{TMTSF})_{2}\left(\mathrm{AsF}_{6}\right)_{(1-x)}\left(\mathrm{SbF}_{6}\right)_{x}$.

In Fig. 3 we compare the temperature dependence of $\sigma_{n}, \sigma_{\mathrm{SDW}}^{\mathrm{dc}}$, and $\sigma_{\mathrm{SDW}}^{\mathrm{ac}}$. In a limited temperature range $2 \mathrm{~K}<T<4 \mathrm{~K}$, both $\sigma_{\mathrm{SDW}}^{\mathrm{sc}}$ and $\sigma_{\mathrm{SDW}}^{\mathrm{sc}}$ follow the temperature dependence of $\sigma_{n}$. Above $4 \mathrm{~K}, \sigma_{\mathrm{SDW}}$ becomes temperature independent. Below $2 \mathrm{~K}$, the scaling between $\sigma_{n}$ and $\sigma_{\mathrm{SDW}}^{\mathrm{ac}}$ continues to hold, but $\sigma_{\mathrm{SDW}}^{\mathrm{dc}}$ decreases faster than the other two quantities.

The scaling $\sigma_{\mathrm{SDW}} \propto \sigma_{n}$, also found in CDW systems [10], is well understood [11]: at the origin of the DW damping in this region is the Coulomb interaction between the DW and the thermally excited quasiparticles. Because of the random pinning, an electric field induces inhomogeneous phase deformations, i.e., also charge fluctuations in the DW. These charge fluctuations are screened by the normal electrons. If the screening is complete, i.e., the temperature is not too low and the frequency is not too high, the dissipation by the screening currents leads to a damping proportional to the resistivity of normal electrons $\sigma_{n}^{-1}$.

Below a crossover temperature $T_{x \mathrm{l}} \approx 2 \mathrm{~K}$, the behavior of $\sigma_{\mathrm{SDW}}^{\mathrm{do}}$ changes abruptly: at low temperatures $\sigma_{\mathrm{SDW}}^{\mathrm{dc}}$ decreases faster than $\sigma_{n}$, consequently the nonlinearity of the current-voltage characteristics disappears. This crossover is not accompanied by any change in the threshold field or in the static dielectric constant [12]: both quantities are constant between 1.5 and $4 \mathrm{~K}$. Neither is any change in the magnetic structure of the SDW as seen by NMR [13] and $\mu$ SR [14] measurements.

The fact that no change is observed in the behavior of the dielectric relaxation at the temperature of the crossover in $\sigma_{\mathrm{SDW}}^{\mathrm{ac}}$, moreover, the normal conductivity at $T_{x 1}$ is as high as $5 \mathrm{~S} / \mathrm{cm}$ (i.e., only two orders of magnitude smaller than at room temperature) rules out the possibility that the "freezing out" of the nonlinearity is due to the freezing out of the normal conductivity. We propose instead-based on the different behavior of $\sigma_{\text {SDW }}^{\mathrm{dc}}$ and $\sigma_{\mathrm{SDW}}^{\mathrm{ac}}$ below $T_{\mathrm{xi}}$ - that the system becomes spatially inhomogeneous at low temperatures. If a small fraction $\eta$ of the sample volume is strongly pinned, the dielectric constant is multiplied by $1-\eta$ only, but because of the very anisotropic nature of the SDW sliding, the nonlinear conductivity may be much more strongly influenced.

At $T_{x 2} \approx 4.5 \mathrm{~K}$ another crossover occurs in the SDW conductivity: above $T_{x 2}, \sigma_{\text {SDW }}$ is temperature independent. $\sigma_{\mathrm{SDW}}$ in the temperature-independent region decreases fast with increasing defect concentration, and in the same time $T_{x 2}$ shifts to lower temperatures. This behavior suggests that at high temperatures, where the damping by the quasiparticle screening currents is small, another mechanism-determined by the SDW-defect interaction-takes over. In Fig. 4 we compare the high- 


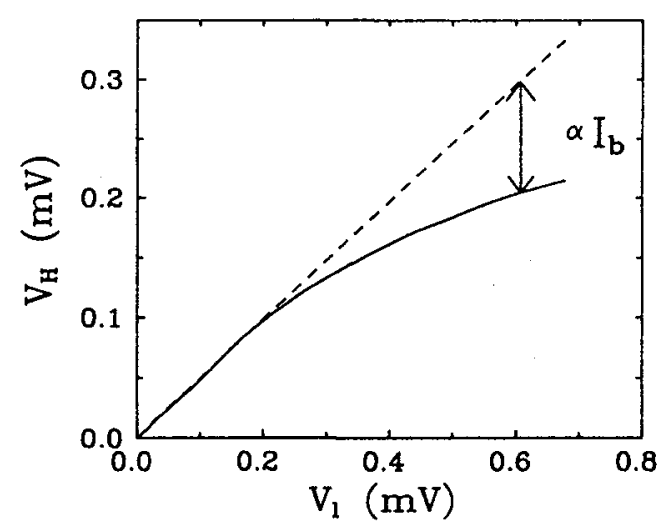

FIG. 5 Hall voltage $V_{H}$ as a function of the longitudinal voltage $V_{l}$ in (TMTSF) ${ }_{2} \mathrm{PF}_{6}$. The nonlinear relation above the depinning threshold indicates the breakdown of the noninteracting two-fluid model. The deviation from the linear extrapolation of $V_{H}$ is proportional to the backflow of normal carriers, $I_{b}$ in the model described by Eqs. (2).

temperature SDW conductivity to the depinning threshold in the series (TMTSF $)_{2}\left(\mathrm{AsF}_{6}\right)_{(1-x)}\left(\mathrm{SbF}_{6}\right)_{x}$; within error, $\sigma_{\mathrm{SDW}}$ scales with $E_{T}$. The overall temperature dependence of the damping $\gamma$ is then written as

$$
\gamma=\alpha E_{T}+\beta \sigma_{n}^{-1}
$$

where $\alpha$ and $\beta$ are independent of temperature and defect concentration. For $T>T_{x 2}$ the first term, for $T>$ $T_{x 2}$ the second term dominates.

\section{Nonlinear quasiparticle transport}

It is often assumed in the analysis of transport measurements that the transport coefficients of the thermally excited normal electrons are the same in the pinned and depinned DW state. This assumption can be tested by the examination of transport coefficients to which the DW does not contribute. No DW contribution is expected for example to the Hall effect because of the strictly one-dimensional nature of the density wave transport, or to the Seebeck and Peltier effects, as the entropy transport by the DW is negligible. The experiments clearly indicate in all the three above cases (Refs. [15,16,17], respectively) that the transport coefficients do become nonlinear when the DW is depinned.

In Fig. 5 we demonstrate the nonlinear quasiparticle transport by Hall effect data [18] in the SDW state of (TMTSF) ${ }_{2} \mathrm{PF}_{6}$. As the Hall voltage $V_{H}$ is proportional to the normal current, the nonlinear $V_{H}$ vs. $V$ curve indicates that the normal current also becomes nonlinear when the SDW is depinned.

The simplest system of equations that accounts for the phenomenon is the following:

$$
\begin{aligned}
& \mathbf{j}_{\mathbf{n}}=\sigma_{n} \mathbf{E}+\frac{\sigma_{n}}{n_{n} e} \mathbf{j}_{\mathbf{n}} \times \mathbf{B}-\alpha j_{\mathrm{DW}} \hat{\mathbf{x}} \\
& j_{\mathrm{DW}}=\sigma_{\mathrm{DW}} E-\beta j_{x}^{n} \\
& \mathbf{j}=\mathbf{j}_{\mathbf{n}}+\mathrm{j}_{\mathrm{DW}} \hat{\mathbf{x}}
\end{aligned}
$$

Here $n_{n}$ is the density of normal electrons, $\mathbf{B}$ is the magnetic field, and the density wave slides in the $x$ direction. The normal conductivity is taken isotropic for simplicity. The last terms in Eqs. (2a) and (2b) describe the interaction between the sliding density wave and the normal current: the DW current induces a backflow of quasiparticles and vice versa. In the Hall effect measurements the current is forced along the $x$ direction, and the transverse field $E_{y}$ is measured with $B$ perpendicular to the $x-y$ plane. From Eqs. (2) we obtain

$$
E_{y}=(B / n e) j_{x}^{n}=(B / n e)\left(\sigma_{n} E_{x}-\alpha j_{\mathrm{DW}}\right),
$$


which is nonlinear, as illustrated in Fig. 5 , because of the nonlinearity of $j_{\mathrm{Dw}}$. The data of Fig. 5 are well described by an electric-field-independent backflow coefficient $\alpha=0.2$.

A system of equations similar to Eqs. (2) has been first set up and investigated by Boriack and Overhauser [19] for metallic CDW systems where the Peierls transition removes a small part of the Fermi surface only. Later Artemenko and Kruglov [20] revisited the problem more carefully and predicted a positive $\alpha$ in Eq. (2a) for insulating CDW's. Their logic is briefly the following: If we consider a DW sliding with a velocity $V$, the quasiparticle spectrum in the sliding frame is the same as the spectrum for a static DW. However, the spectrum in the crystal frame (obtained by Galilei transformation) is different: the gap is displaced in the momentum space and becomes slightly smaller. The situation is different from a semiconductor in which the gap is not at the center of the Brillouin zone, because the sliding density wave breaks the time reversal symmetry, and the quasiparticles at the gap carry a finite momentum, i.e., drift with the DW in real space. In the absence of defect scattering, these drifting quasiparticles do not contribute to the transport coefficients because of the exact electron-hole cancellation. The defect scattering, however, modifies the quasiparticle distribution, and leads to a change in the transport coefficients proportional to the sliding velocity $V$.

The baseline of the above arguments for our discussion is that the change in the quasiparticle drift velocities is in the order of the DW drift velocity $V$. In the following, we show that this effect gives a backflow coefficient orders of magnitude smaller than observed in the experiments. In a typical experiment, the current carried by the DW is of the same order of magnitude as the quasiparticle current, but the density of condensed electrons is much higher than the density of normal electrons. In our example, (TMTSF) ${ }_{2} \mathrm{PF}_{6}$ at $T=4.2 \mathrm{~K}$, Hall effect measurements give a normal electron concentration $n_{n} \sim 10^{-4} n$, where $n$ is the total electron density. This value is in good agreement with the density calculated using a gap $\Delta=20 \mathrm{~K}$. The fact that despite their low concentration, the quasiparticles carry the same current as the $\mathrm{DW}$ indicates that the quasiparticle drift velocity is 4 orders of magnitude higher than the sliding velocity. Therefore the backflow coefficient from the Artemenko-Kruglov mechanism is in the order of $10^{-4}$, in contrast to the observed $\alpha \sim 0.1$. The discrepancy is even more serious in magnetic-field-induced spindensity waves where $\alpha \approx 0.8$ has been obtained [21].

It is easy to see that the Coulomb interaction between the SDW and the normal electrons-not considered by Artemenko and Kruglov-may also lead to quasiparticle backflow. If a SDW charge packet propagates along the conducting chains (e.g. in form of a propagating SDW dislocation loop), it carries along the screening normal electrons which appears as a backflow in transport measurements. If the spatial separation of these charge packets is large, this mechanism can give a backflow coefficient close to 1 .

\section{Conclusion}

We have shown, that the pinning of the SDW is strongly enhanced by a large concentration of weakly perturbing nonmagnetic point defects.

The damping of the low-frequency phase mode shows a crossover from a low-temperature region where the damping is determined by the quasiparticle screening currents to a high-temperature region where the damping is dominated by the SDW-defect interaction. The temperature dependence of the crossover together with magnitude of the high-temperature damping suggest that the crossover results from the different temperature dependence of the two mechanisms and not from a phase transition $[22,23]$ in the SDW. We believe that the same crossover in phason dynamics is reflected in the crossover in the NMR spin-lattice relaxation rate $[22,24]$. We note that no theory explains the observed temperatureindependent damping; the only theoretical study [25] of the effect of defect scattering on the damping predicts $\gamma \propto \exp (-\Delta / T)$.

Hall effect measurements indicate that the quasiparticle transport also becomes strongly nonlinear when the SDW is depinned. This nonlinearity is interpreted in an interacting two-fluid model. The measured coupling between the SDW and normal electrons, however, is much larger than obtained from the kinetic theory of Artemenko and Kruglov [20]. Coulomb effects may enhance the backflow coefficient.

We are grateful to C. Lenoir, Y.-S. Huang, and P. Batail for preparing the samples. Useful discussions with $\mathrm{H}$. J. Schulz are acknowledged. 


\section{References}

[a] Also at Research Institiute for Solid State Physics, P.O.B. 49, H-1525 Budapest, Hungary.

[b] Present address: Hydro Aluminium a.s Karmøy, N-4265 Håvik, Norway.

[1] For a recent review on charge-density waves, see Charge Density Waves in Solids, edited by L. P. Gor'kov and G. Grüner (North-Holland, Amsterdam, 1989).

[2] For a collection of recent papers on spin-density waves, see Proceedings of the International Conference on Science and Technology of Synthetic Metals, Göteborg, Sweden, 1992, Synth. Metals Vols. 55-57 (1993).

[3] I. Tüttö and A. Zawadowski, Phys. Rev. Lett. 60, 1442 (1988); K. Maki and A. Virosztek, Phys. Rev. B 39, 9640 (1989).

[4] W. Kang, S. Tomic, and D. Jérome, Phys. Rev. B 43, 1264 (1991).

[5] 0 . Traetteberg et al., to be published.

[6] O. Traetteberg, Ph.D. Thesis, Université Paris-Sud, Orsay, 1993.

[7] L. Zuppiroli and S. Bouffard, J. Phys. (France) 41, 1961 (1980).

[8] S. Tomic, J. R. Cooper, W. Kang, D. Jérome, and K. Maki, J. Phys. (France) I 1, 1603 (1991).

[9] K. Maki and A. Virosztek, Phys. Rev. B 42, 655 (1990).

[10] R. M. Fleming, R. J. Cava, L. F. Schneemeyer, E. A. Rietman, and R. G. Dunn, Phys. Rev. B 33, $5450(1985)$.

[11] P. B. Littlewood, Phys. Rev. B 36, 3108 (1987).

[12] O. Traetteberg and G. Kriza, to be published.

[13] W. G. Clark et al., this conference.

[14] Y. Uemura, private communication.

[15] Yu. I. Latyshev, Ya. S. Savitskaya, and V. V. Frolov, Proceeding of the International Conference on Charge Density Woves in Solids, Budapest, Hungary, 1984, edited by Gy. Hutiray and J. Sólyom, Vol. 217 of Lecture Notes in Physics (Springer, Berlin, 1985), p.339.

[16] G. Mihály, G. Kriza, and G. Grüner, Solid State Commun. 68, 993 (1988).

[17] G. Kriza, L. Forró, and A. Jánossy, Phys. Rev. B 41, 5451 (1990).

[18] O. Traetteberg, L. Balicas, and G. Kriza, this conference.

[19] M. L. Boriack and A. W. Overhauser, Phys. Rev. B 16, 5206 (1977); ibid., p.5256.

[20] S. N. Artemenko and A. N. Kruglov, Fiz. Tverd Tela 26, 2391 (1984).

[21] L. Balicas, N. Biskup, and G. Kriza, this conference.

[22] T. Takahashi et al., Physica 143B, 417 (1986).

[23] J. C. Lasjaunias, K. Biljakovic, P. Monceau, and K. Bechgaard, Solid State Commun. 84, 297 (1992), and this conference.

[24] W. H. Wong, M. E. Hanson, B. Alavi, W. G. Clark, and W. A. Hines, Phys. Rev. Lett. 70, 1882 (1993).

[25] K. Maki and A. Virosztek, Phys. Rev. B 39, 2511 (1989). 